\title{
Standard Hydrogen Test Protocols for the NREL Sensor Testing Laboratory
}

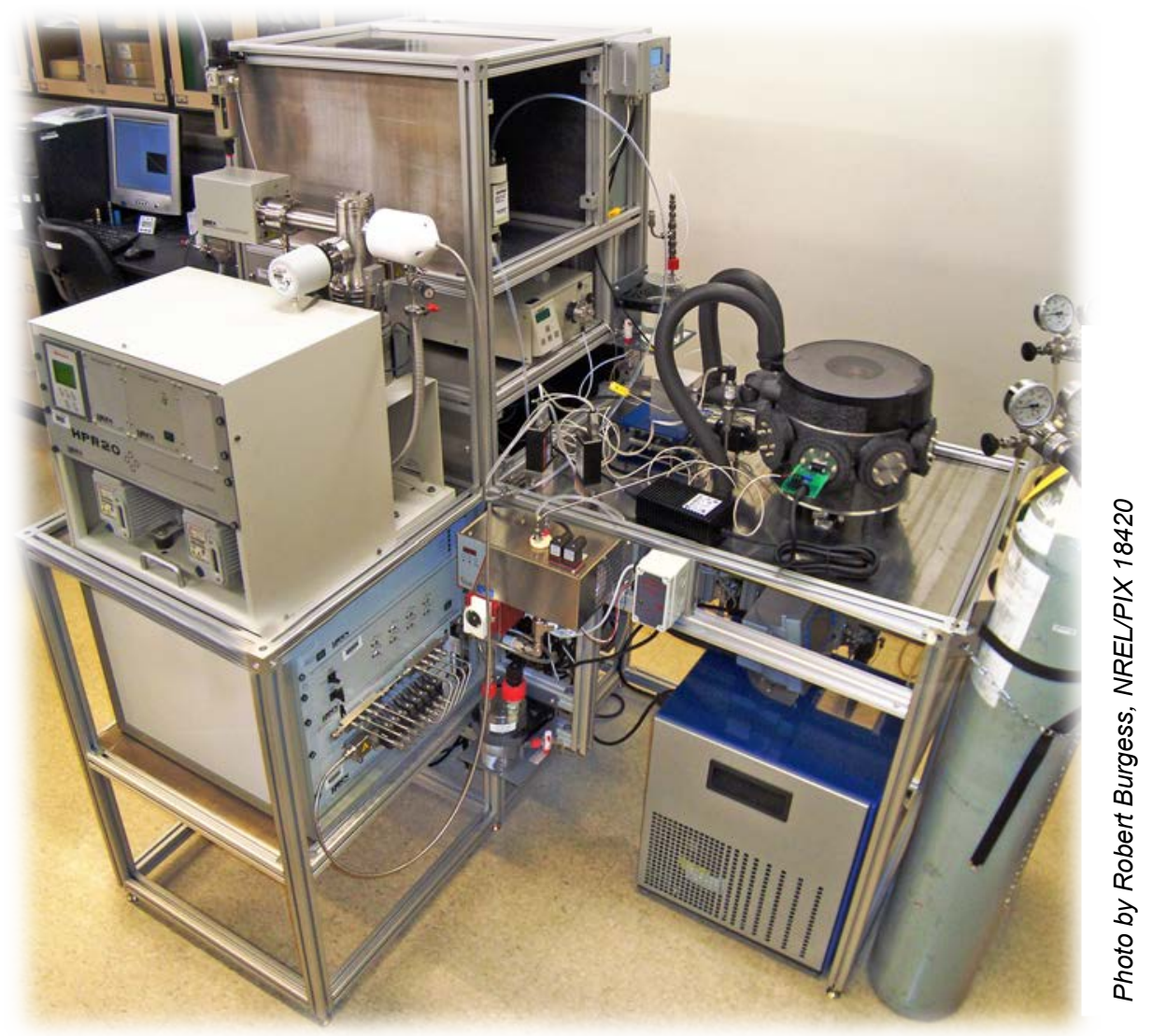

\section{Codes\& Safety Standards}

December 2011

NREL is a national laboratory of the U.S. Department of Energy, Office of Energy Efficiency and Renewable Energy, operated by the Alliance for Sustainable Energy, LLC. 



\section{Standard Test Protocols for the NREL Hydrogen Sensor Test Laboratory}

Researchers at the NREL Hydrogen Safety Sensor Test Laboratory ${ }^{1}$ developed a variety of test protocols to quantitatively assess critical analytical performance specifications for hydrogen sensors. Many are similar to, but typically more rigorous than, the test procedures mandated by ISO Standard 26142 (Hydrogen Detector for Stationary Applications). Specific protocols were developed for linear range, short-term stability, and the impact of fluctuations in temperature $(T)$, pressure $(P)$, relative humidity $(\mathrm{RH})$, and chemical environment. Specialized tests (e.g., oxygen requirement) may also be performed.

Hydrogen safety sensors selected for evaluation are subjected to a thorough regimen of test protocols, as described below. Sensor testing is performed at NREL on custom-built sensor test fixtures ${ }^{2}$. Environmental parameters such as T, P, RH, and gas composition are rigorously controlled and monitored. The NREL evaluations are performed on commercial hydrogen detectors, on emerging sensing technologies, and for end users to validate sensor performance for specific application needs. Test results and data are shared with the manufacturer or client via summary reports, teleconference phone calls, and, when appropriate, site visits to manufacturer facilities. Client representatives may also monitor NREL's operation while their technologies are being tested. Manufacturers may use test data to illustrate the analytical capability of their technologies and, more importantly, to guide future developments. NREL uses the data to assess technology gaps and deployment considerations. Per NREL Sensor Testing Laboratory policy, test results are treated as proprietary and are not shared with other manufacturers or other entities without permission. The data may be used by NREL in open publications (journal articles, presentations, outreach support, and other reports), but will not be attributed to a specific vendor.

The following sections describe standard test protocols developed by the NREL sensor test laboratory. Modifications are implemented based on restrictions imposed by the technology type or requirements of the client. In this protocol discussion, nominal test conditions adhere to the following typical tolerances:

- The test gas concentrations are mixed within $\pm 10 \%$ of the nominal concentration, but are known to within $\pm 2 \%$. Test gases are generated from blending certified gas mixtures with synthetic air.

- The typical test range is $0 \%-2 \%$ hydrogen in air. The concentration range can be extended to $8 \%$ hydrogen in $20 \%$ air. For low-range sensors, the test gas concentrations can be adjusted to $0 \%$ $0.2 \%$. Hydrogen concentrations can be reliably generated as low as $0.005 \%$.

- The sensor test chamber is purged at a regulated gas flow rate that is maintained to within $\pm 5 \%$ of the nominal flow rate.

- The temperature tolerance is typically $\pm 2^{\circ} \mathrm{C}$ of the nominal temperature.

- The RH tolerance is typically $\pm 5 \%$ of the nominal $\mathrm{RH}$ set point. Humidity can be reported as RH or dew point.

- The pressure tolerance is typically \pm 0.05 bar of the nominal pressure set point.

For more information please contact:

William Buttner - William.Buttner@nrel.gov

Robert Burgess - Robert.Burgess@nrel.gov

Matthew Post - Matthew.Post@nrel.gov

Carl Rivkin-Carl.Rivkin@nrel.gov

\footnotetext{
${ }^{1}$ See http://www.nrel.gov/hydrogen/facilities_hsl.html

${ }^{2} \mathrm{http}: / / \mathrm{www} . n r e l . g o v / h y d r o g e n / p d f s / 42987 . \overline{p d f}$
} 


\section{Sensor Calibration Test/Validation Test}

The sensor Calibration Test/Validation Test subjects the sensor to exposures of two concentrations of hydrogen in air in the sequence illustrated in Figure 1. Gas concentrations are each obtained from certified gas cylinders of $1 \%$ hydrogen in air and $2 \%$ hydrogen in air. The allowable tolerance of each test gas is to within $\pm 10 \%$ of the nominal concentration, but the actual concentration is known to within $\pm 2 \%$. The duration of each step is typically one hour, but may be adjusted to allow the instrument to reach a stable, steady-state response (e.g., the "final indication"). A minimum 10-minute exposure time is required once the instrument has stabilized to its final indication. The Sensor Calibration Test is typically performed prior to (called the "Initial Calibration) and following (called the "Final Calibration") all other tests, including many of those listed below. No instrument adjustment is allowed between the Initial Calibration and the Final Calibration. This requirement allows for an assessment of the impact of the various test protocols on sensor stability. The Sensor Calibration Test is performed under the following conditions:

Temperature:

Pressure:

Relative Humidity:

Gas Flow Rate: $25^{\circ} \pm 2^{\circ} \mathrm{C}$

$1.0 \pm 0.05$ bar

$50 \% \pm 5 \%$ (dew point approximately $12^{\circ}-13.8^{\circ} \mathrm{C}$ )

$1000 \pm 20 \mathrm{sccm}$

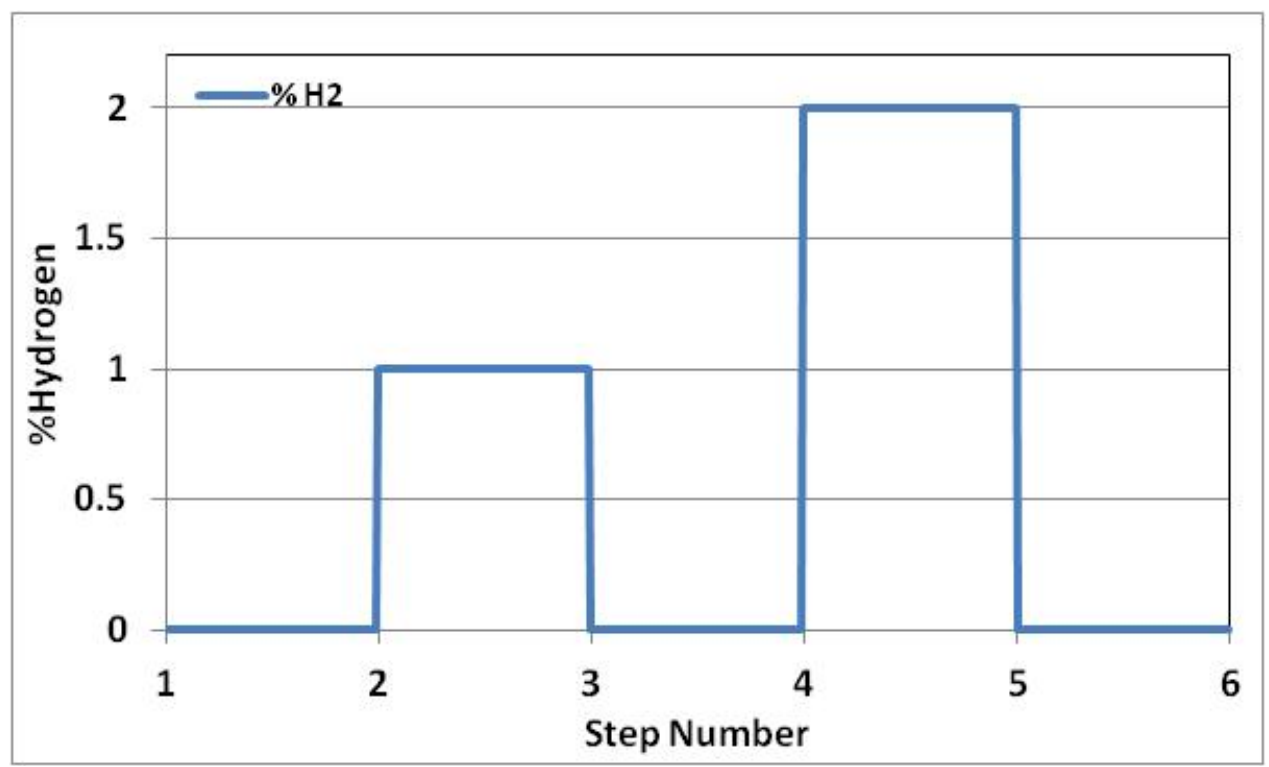

Figure 1: The Sensor Calibration/Sensor Validation Test. Sensors are exposed to $1 \%$ and to $2 \%$ hydrogen in air; each test gas is obtained from certified gas standards. The sensors are allowed to recover in air following test gas exposure. The test can be performed several times, typically prior to (the "Initial Calibration") and following (the "Final Calibration") subjecting the sensor to other test protocols. The test is performed to investigate the impact of the various test protocols on sensor performance. 


\section{Short Term Stability Test/Repeatability Test}

The sensor is subjected to exposure cycles that comprise $0.0 \%, 0.2 \%, 1.0 \%$, and $2.0 \%$ hydrogen at a constant flow rate of $1000 \mathrm{sccm}$, as illustrated in Figure 2. The concentration range can be adjusted for low-range sensors (e.g., $0.0 \%, 0.05 \%, 0.1 \%$, and $0.2 \%$ hydrogen). The duration of each step is typically one hour, but may be set to allow the instrument to reach a stable, steady-state response (i.e., the "final indication"). A minimum 10-minute exposure time is required once the instrument has stabilized to its final indication. The test sequence shown in Figure 2 is performed nine times over a three-day period to assess short-term signal stability (additional time may be allowed if the test is interrupted by nonbusiness days or other events). The Short Term Stability Test is performed under the following conditions:

Temperature:

Pressure:

Relative Humidity:

Gas Flow Rate: $25^{\circ} \pm 2^{\circ} \mathrm{C}$

$1.0 \pm 0.05$ bar

$50 \% \pm 5 \%$ (dew point approximately $12^{\circ}-13.8^{\circ} \mathrm{C}$ )

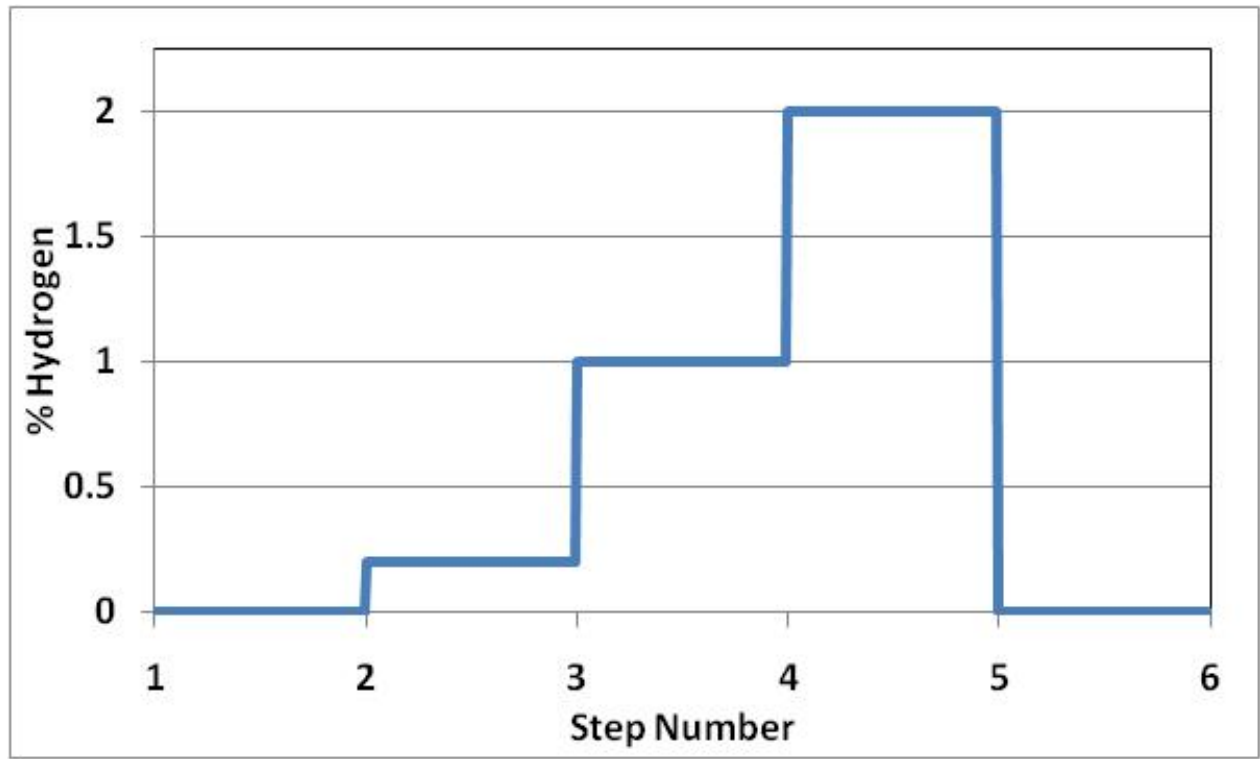

Figure 2: Short Term Stability Test/Repeatability Test protocol. Each sensor is sequentially exposed to $0.0 \%, 0.2 \%, 1.0 \%, 2.0 \%$, and $0.0 \%$ hydrogen in air. This sequence is performed nine times over three days. The duration of each step is set by the time required for the sensor to reach a stable signal. 


\section{Accuracy of Response (Linear Range Test)}

In the Accuracy of Response Test (Linear Range Test), sensors are exposed to a gas mixture whose hydrogen concentration is changed stepwise between $0 \%$ and $2 \%$ hydrogen (maximum) by online mixing of $2 \%$ hydrogen in air with synthetic air. The aim of the Accuracy of Response Test is to determine the accuracy of the sensor output to changes in hydrogen concentration as validated by a comparison with independent gas analysis. Typically the instrument is tested using calibration parameters from the manufacturer; NREL can, however, perform on-site calibrations. The gas composition is set by the relative flow rates of mass flow controllers (MFCs). The change in hydrogen concentration is performed by increasing and then decreasing the relative ratio of $2 \%$ hydrogen and synthetic air in defined sequential steps, as illustrated in Figure 3 . The specific hydrogen concentrations are $0.0 \%, 0.2 \%, 0.4 \%$, $0.8 \%, 1.2 \%, 1.6 \%, 2.0 \%, 1.6 \%, 1.2 \%, 0.8 \%, 0.4 \%, 0.2 \%$, and $0.0 \%$. The sensors signals are monitored to ensure a stable response. Gas concentration may be monitored by a mass spectrometer to ensure a stable, steady-state condition in the sensor test chamber. Sensor outputs and the corresponding measured hydrogen concentration are noted and compared to assess the accuracy of the sensor response. Typical test conditions for the accuracy tests are:
Temperature:
$25^{\circ} \pm 2^{\circ} \mathrm{C}$
Pressure:
$1.0 \pm 0.05$ bar
Relative Humidity:
$50 \% \pm 5 \%$
Gas Flow rate:
$1000 \pm 20 \mathrm{sccm}$

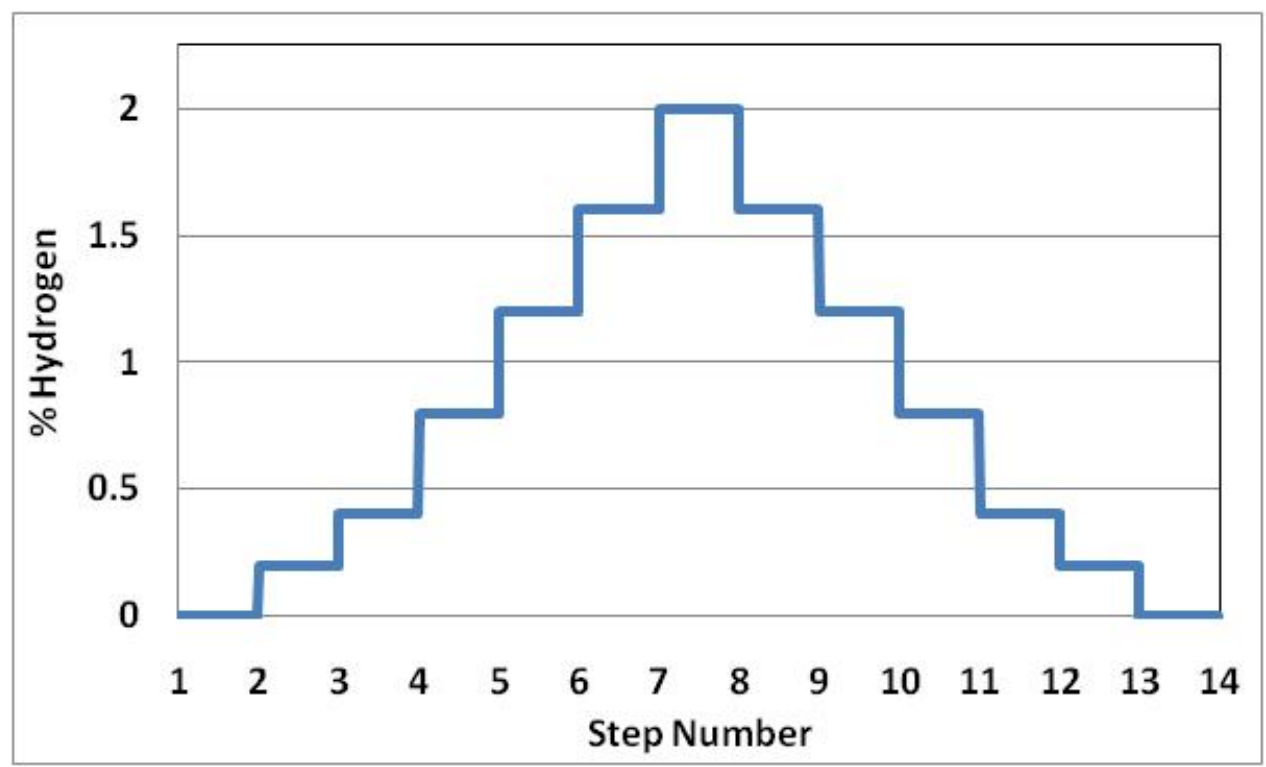

Figure 3: Accuracy of Response Test (Linear Range) protocol. Each sensor is sequentially exposed to $0.0 \%, 0.2 \%, 0.4 \%, 0.8 \%, 1.2 \%, 1.6 \%, 2.0 \%, 1.6 \%, 1.2 \%, 0.8 \%, 0.4 \%, 0.2 \%$, and $0.0 \%$ hydrogen in air. The duration of each step is typically one hour, but may be set by the time required for the sensor to reach a stable signal.

The results from the Accuracy of Response Test can be used to determine the sensor lower detection limit, determination limit, linear range, analytical resolution, and sensitivity. 


\section{Ambient Pressure}

The aim of the Pressure Test is to determine the influence of pressure on the sensor output signal in the absence and presence of hydrogen in concentrations up to $2 \%$ hydrogen in air. The pressure in the test chamber is set to at least three different pressures in the range of 0.8-1.2 bar, unless outside of the manufacturer pressure specifications. The range of the pressure tests may be extended from 0.4 to 1.2 bar. The default pressure set points are $0.8,1.0$, and 1.2 bar to within \pm 0.05 bar. Chamber pressure is monitored using a certified pressure transducer. For pressure testing, the test fixture and sensor are allowed to stabilize and equilibrate at each pressure set point. Once stabilized, the sensors are exposed to air and then up to $2 \%$ hydrogen in air using an exposure profile that comprises $0.0,0.2,1.0$, and 2.0 vol\% hydrogen in air. The concentration range can be adjusted for low-range sensors (e.g., 0.0\%, 0.05\%, $0.1 \%$, and $0.2 \%$ hydrogen). The exposure profile will include a final exposure to $0 \%$ hydrogen in synthetic air. Upon completion of the hydrogen exposure (including final recovery period in air), the pressure of the test chamber is adjusted to the next pressure set point. The overall exposure profile for the Ambient Pressure Test is illustrated in Figure 4.

All Ambient Pressure Tests are performed under the following conditions:

Temperature: $\quad 25^{\circ} \pm 2^{\circ} \mathrm{C}$

Pressure: $\quad$ Variable to the designated set point \pm 0.05 bar

Relative Humidity: $\quad 50 \% \pm 5 \%$ (dew point approximately $12^{\circ}-13.8^{\circ} \mathrm{C}$ )

Gas Flow Rate: $\quad 1000 \pm 20 \mathrm{sccm}$

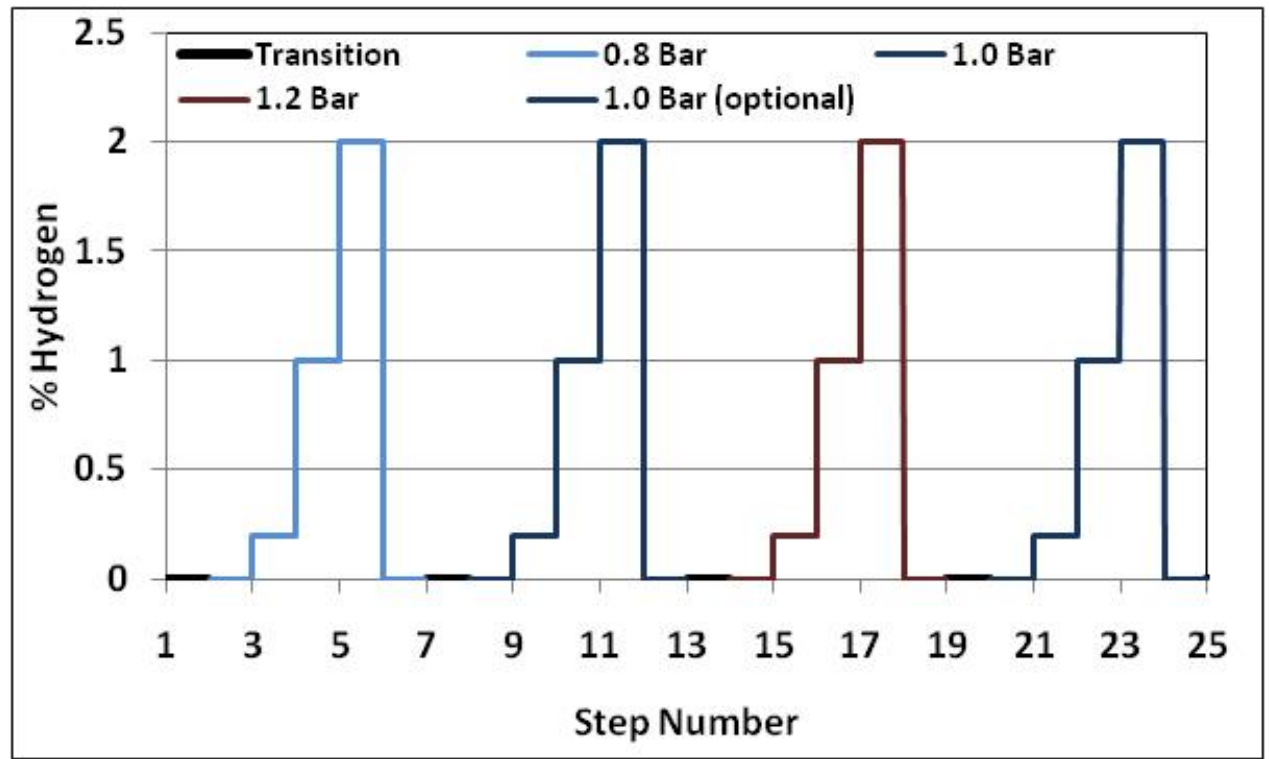

Figure 4: Pressure Dependence Test protocol. Each sensor is sequentially exposed to $0.0 \%, 0.2 \%$, $1.0 \%, 2.0 \%$, and $0.0 \%$ hydrogen in air at each pressure set point, typically $0.8,1.0$, and 1.2 bar (to within \pm 0.05 bar). The duration of each step is set by the time required for the sensor to reach a stable signal. 


\section{Ambient Temperature}

The aim of the Ambient Temperature Test is to determine the influence of temperature on the sensor output signal in the absence and presence of hydrogen in concentrations up to $2 \%$ hydrogen in air. The temperature in the test chamber is set to up to five different temperatures that encompass the temperature range specified by the sensor manufacturer. Unless outside of the manufacturer specifications, the temperature set points are $-20^{\circ}, 0^{\circ}, 25^{\circ}, 50^{\circ}$, and $85^{\circ} \mathrm{C}$. Chamber temperature is monitored using a certified temperature transducer. The test fixture and sensor are allowed to stabilize and equilibrate at each temperature set point prior to hydrogen exposure. Once stabilized, the sensors are exposed to air and then up to $2 \%$ hydrogen in air using an exposure profile that comprises $0.0,0.2$, 1.0 , and 2.0 vol\% hydrogen in air (depending on the range of the sensor being tested). The concentration range can be adjusted for low-range sensors (e.g., $0.0 \%, 0.05 \%, 0.1 \%$, and $0.2 \%$ hydrogen). The exposure profile will include a final exposure to $0 \%$ hydrogen in synthetic air. Upon completion of the hydrogen exposure (including final recovery period), the temperature of the test chamber is adjusted to the next temperature set point. The overall exposure profile for the Ambient Temperature Test is illustrated in Figure 5. All Ambient Temperature Tests are performed under the following conditions:

Temperature:

Pressure:

Relative Humidity:

Gas Flow Rate:
Variable to the designated set point $\pm 2^{\circ} \mathrm{C}$

$$
1.0 \pm 0.05 \mathrm{bar}
$$$$
<5 \% \text { (at } 25^{\circ} \mathrm{C} \text { ) }
$$

$1000 \pm 20 \mathrm{sccm}$

The temperature test is performed using dry gas, with a dew point less than the lowest temperature set point.

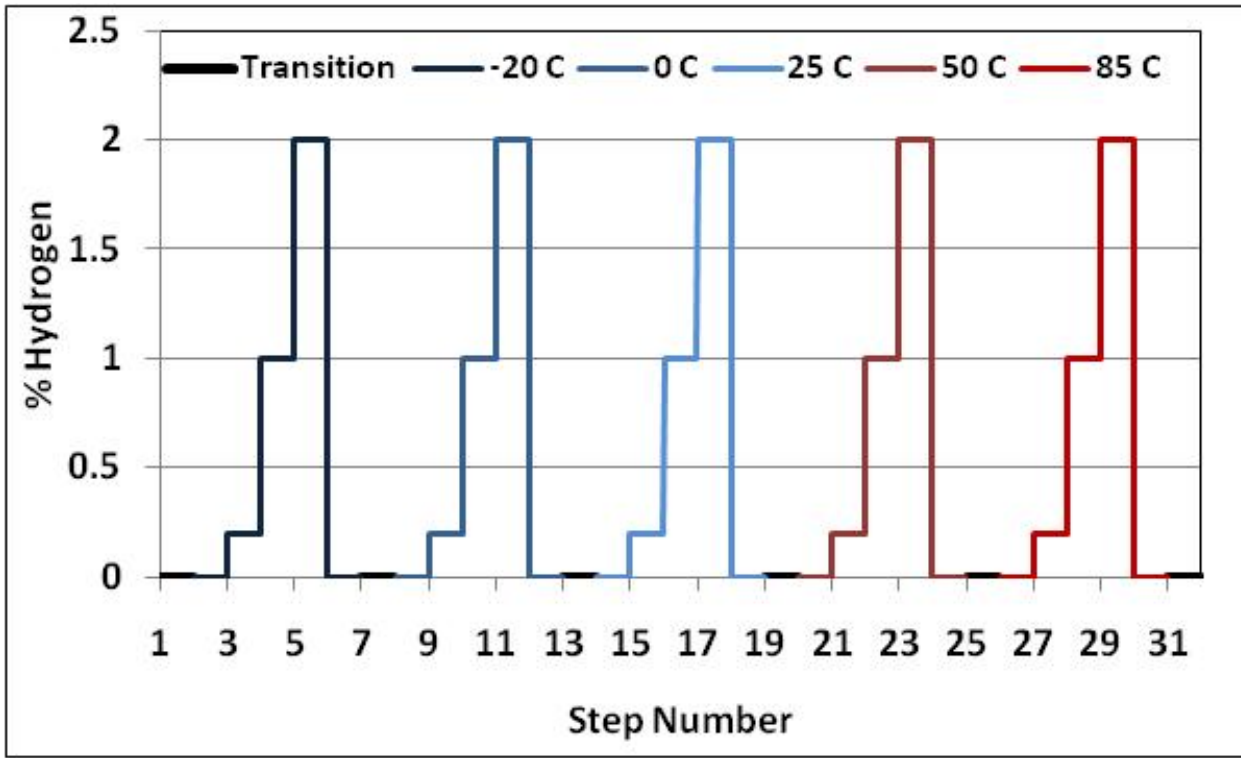

Figure 5: Temperature Dependence Test protocol. Each sensor is sequentially exposed to $0.0 \%$, $0.2 \%, 1.0 \%, 2.0 \%$, and $0.0 \%$ hydrogen in air at each temperature set point, typically $-20^{\circ}, 0^{\circ}, 25^{\circ}$, $50^{\circ}$, and $80^{\circ} \mathrm{C}$ (to within $\pm 2^{\circ} \mathrm{C}$ ). The duration of each step is typically one hour, but may be set by the time required for the sensor to reach a stable signal. 


\section{Ambient Humidity Test}

The aim of the Ambient Humidity Test is to determine the influence of moisture fluctuations on the sensor output signal in the absence and presence of hydrogen in concentrations up to $2 \%$ hydrogen in air. The humidity test is performed at $25.0^{\circ} \pm 2.0^{\circ} \mathrm{C}$ and a barometric pressure of $1.0 \pm 0.05$ bar. The RH set points are dry $(<5 \% \mathrm{RH}), 25 \% \pm 5 \%, 50 \% \pm 5 \%$, and $85 \% \pm 5 \%$. Chamber $\mathrm{RH}$ is monitored using a certified RH transducer. Other RH set points may be used. Humidity can be reported as RH (measured) or as dew point (calculated as per August-Roche-Magnus Approximation ${ }^{3}$ ). For ambient humidity testing, the test fixture is allowed to stabilize at each humidity set point. Once the test chamber reaches a stable humidity, the sensor is allowed to stabilize at least 15 minutes before hydrogen exposure tests are initiated. Once stabilized, the sensors are exposed to synthetic air and then up to $2 \%$ hydrogen in air using an exposure profile that comprises $0.0,0.2,1.0$, and 2.0 vol\% hydrogen in air. The concentration range can be adjusted for low-range sensors (e.g., $0.0 \%, 0.05 \%, 0.1 \%$, and $0.2 \%$ hydrogen). The exposure profile will include a final exposure to $0 \%$ hydrogen in synthetic air. Upon completion of the hydrogen exposure (including final recovery period), the humidity of the test chamber is adjusted to the next humidity set point. The overall exposure profile for the Ambient Humidity Test is illustrated in Figure 6. All tests are performed under the following conditions:
Temperature:
$25^{\circ} \pm 2^{\circ} \mathrm{C}$
Pressure:
$1.0 \pm 0.05$ bar
Relative Humidity:
Variable to the designated set point $\pm 5 \%$
Gas Flow Rate:
$1000 \pm 20 \mathrm{sccm}$

A final test sequence performed at $40^{\circ} \mathrm{C}$ and $85 \% \mathrm{RH}$ may be performed.

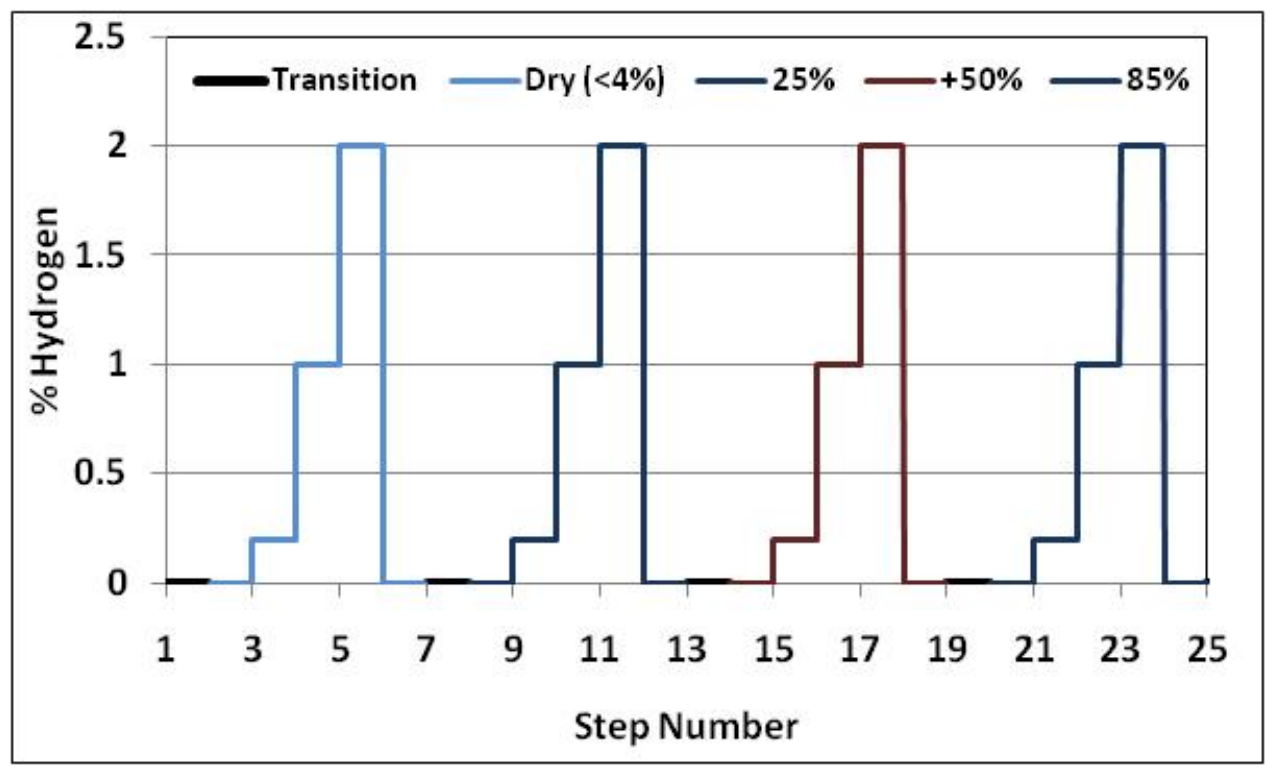

Figure 6: Humidity Dependence Test protocol. Each sensor is sequentially exposed to $0.0 \%, 0.2 \%$, $1.0 \%, 2.0 \%$, and $0.0 \%$ hydrogen in air at each pressure set point, typically dry, $25 \%, 50 \%$, and $85 \%$ $\mathrm{RH}$ (to within $\pm 5 \mathrm{RH}$ units). The duration of each step is set by the time required for the sensor to reach a stable signal.

\footnotetext{
${ }^{3}$ Hardy B. (1998). "ITS-90 Formulations for Vapor Pressure, Frostpoint Temperature, Dewpoint Temperature, and Enhancement Factors in the Range -100 to +100 C." In Proceedings of the Third international Symposium on Humidity \& Moisture, Teddington, London, England, April 1998. www.rhsystems.net/papers/its90form.pdf.
} 


\section{Interferent/Cross Sensitivity Test}

The aim of the Interferent/Cross Sensitivity Test is to determine the selectivity factors for the designated sensor to various interferences. The effect of the interferent in air and the effect of the interferent in air plus $1 \%$ hydrogen are measured. The exposure sequence will consist of a measurement of the sensor response to $1 \%$ hydrogen (performed twice) followed by two or more exposures of the interferent at a predetermined concentration (see Table 1). The sensor is exposed to clean air between each exposure. The exposure time to $1 \%$ hydrogen in air and recovery in air is typically one hour, but may be set by the time required for the sensor to reach a stable, steady-state response, which is based on the sensor/test system response factors. This duty cycle (composed of exposure time to the test gas and recovery time) will remain fixed for the test duration and will define the exposure duty cycle associated with the various interferent gases. Proposed interferents are listed in Table 1. The listed concentrations correspond approximately to Occupational Safety and Health Administration permissible exposure limits or, in the case of methane, the lower flammable limit (LFL). Thus, selectivity coefficients are typically calculated using equivalent hydrogen response induced by the regulated concentration (or fractions thereof) for the specific test gas. Test concentrations are proposed to be at $50 \%$ of this value to allow the test to be performed in air and in $1 \%$ hydrogen in air.

The sensor is subjected to two exposure cycles of the interferent gas at the test concentration. Following the second interferent exposure and recovery in air, the sensor is exposed continuously to $1 \%$ hydrogen while the interferent exposure cycles are repeated two or more times. The concentration of the interferent is not changed. Following the second interferent exposure, the sensor is allowed to stabilize in $1 \%$ hydrogen followed by a recovery time in air. Following the recovery in air, the sensor response to $1 \%$ hydrogen in air is measured twice. Some of the proposed interferents may be poisons (i.e., vapors that induce irreversible effects on sensor behavior). All Cross Sensitivity Tests are performed under the following conditions:
Temperature:
Variable to the designated set point $\pm 2^{\circ} \mathrm{C}$
Pressure:
$1.0 \pm 0.05$ bar
Relative Humidity:
$<5 \%$ (dry)
Gas Flow Rate:
$1000 \pm 20 \mathrm{sccm}$

Table 1: Interferent Test Gases and Concentrations (to within $\pm 10 \%$ )

\begin{tabular}{|l|l|l|l|}
\hline Interferent & \multicolumn{1}{|c|}{$\begin{array}{c}\text { Cylinder } \\
\text { Concentration }\end{array}$} & \multicolumn{1}{|c|}{$\begin{array}{c}\text { Test Concentration } \\
\text { (in Air) }\end{array}$} & \multicolumn{1}{|c|}{$\begin{array}{c}\text { Test Concentration } \\
\text { (in 1\% } \mathrm{H}_{2} \text { ) }\end{array}$} \\
\hline Carbon Monoxide & $100 \mathrm{ppm}$ & $50 \mathrm{ppm}$ & $50 \mathrm{ppm}$ \\
\hline Nitrogen Dioxide & $10 \mathrm{ppm}$ & $5 \mathrm{ppm}$ & $5 \mathrm{ppm}$ \\
\hline Hydrogen Sulfide & $40 \mathrm{ppm}$ & $20 \mathrm{ppm}$ & $20 \mathrm{ppm}$ \\
\hline Methane & $2 \%$ & $1 \%$ & $1 \%$ \\
\hline Ammonia & $100 \mathrm{ppm}$ & $50 \mathrm{ppm}$ & $50 \mathrm{ppm}$ \\
\hline Carbon Dioxide & $1 \%$ & $0.5 \%$ & $0.5 \%$ \\
\hline
\end{tabular}




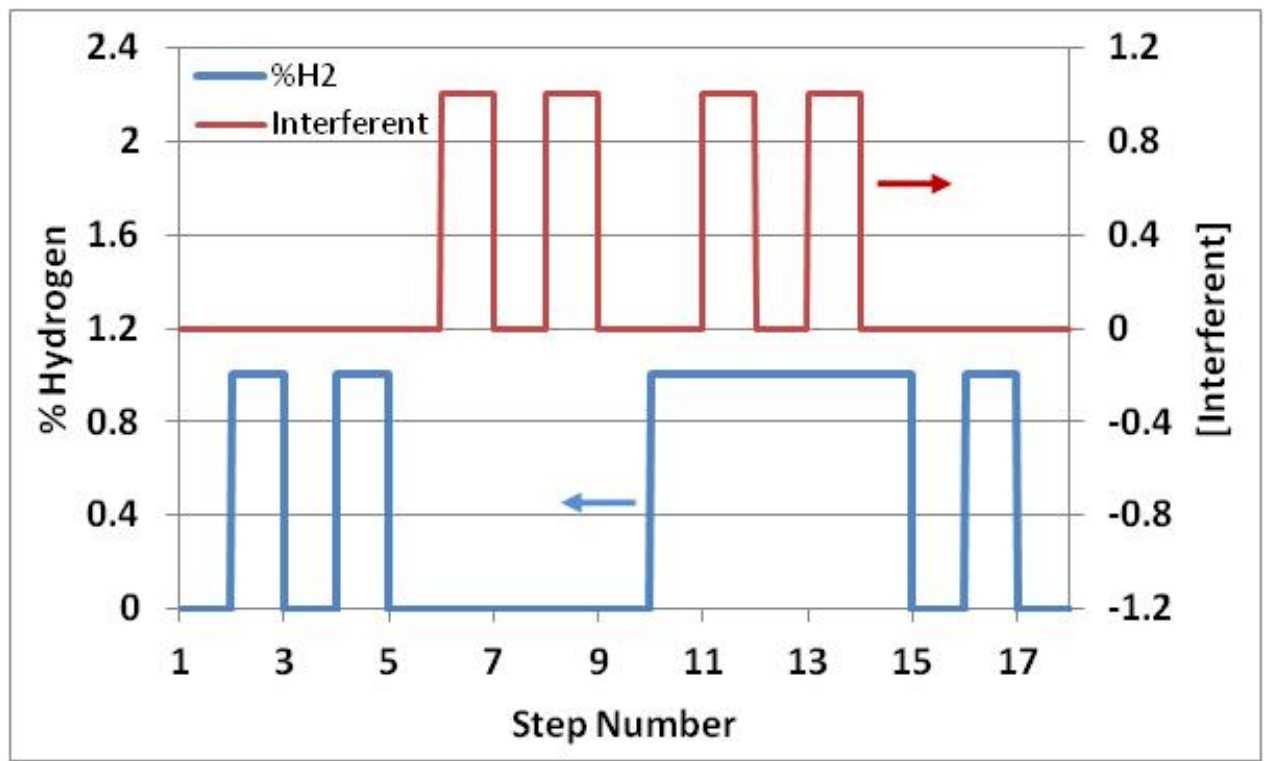

Figure 7: Interferent Test. Each sensor is exposed to $1 \%$ hydrogen in air and then exposed to the interferent gas. The sensor is then exposed to the interferent gas in $1 \%$ hydrogen in air. The duration of each step is one hour or set by the time required for a stable signal. 


\section{Oxygen Dependence Test}

A reliable safety system is composed of various elements that can include intrinsic design features (e.g., pressure control or venting systems), engineering controls (e.g., sample size minimization, removal of oxygen from in or around the system) and hydrogen sensors to monitor for releases. Thus, hydrogen operations are often performed under a depressed oxygen or anaerobic atmosphere to minimize fire and explosion risks. Unfortunately, many sensor platforms are deactivated when operated in an anaerobic atmosphere. Thus, a detector deployed in an anaerobic atmosphere may indicate that no hydrogen is present, but this may not be the case. The nitrogen purge gas could in fact contain high levels of hydrogen and present a dangerous situation when mixed with air upon venting.

To assess sensor performance under depressed oxygen, hydrogen-air and hydrogen-nitrogen mixtures are produced from gas cylinders of air, nitrogen, $1 \%$ or $2 \%$ hydrogen in air, and $1 \%$ or $2 \%$ hydrogen in nitrogen using the gas flow control system of the respective test apparatus. Air is considered here to be $20.9 \%$ oxygen in nitrogen. The air test gas is blended with nitrogen to form gas mixtures with decreased oxygen levels. Specifically, for $50 \%$ air (10.45\% oxygen) blends composed of $50 \%$ air and $50 \%$ nitrogen are used; for $25 \%$ air ( $5.25 \%$ oxygen) blends composed of $25 \%$ air and $75 \%$ nitrogen used; for $0 \%$ air ( $0 \%$ oxygen) pure nitrogen is used. An automated, continuous test protocol was developed that could be demarcated into four distinct sections:

\section{(1). Aerobic Range Section (control)}

The sensor is operated in a normal air atmosphere and is subjected to two sequential exposures of $1 \%$ hydrogen in air. The sensor is allowed to recover in air following each hydrogen exposure.

\section{(2). Oxygen Variation Section}

Following the aerobic range section the sensor is operated in depressed oxygen atmospheres. Nitrogen is used to displace the oxygen. Specific test atmospheres were $50 \%$ air ( $10.45 \%$ oxygen) and $25 \%$ air (5.25\% oxygen). At each atmosphere the sensor is subjected to two sequential exposures of $1 \%$ hydrogen in air/nitrogen mixtures. The sensor is allowed to recover in the test atmosphere following each hydrogen exposure. The aim of this section of the test is to show the integrity of the sensor response to changes in oxygen concentration on the sensor response to $1 \mathrm{vol} \%$ hydrogen and has relevance for the Extended Range Test.

\section{(3). Anaerobic Range Section}

The sensor is operated in an anaerobic atmosphere and is subjected to two sequential exposures of $1 \%$ hydrogen in nitrogen ( $0 \%$ oxygen). The sensor is then exposed to hydrogen concentrations of $0.5 \%$, $0.1 \%$, and $0.05 \%$. The sensor is allowed to recover in nitrogen following the hydrogen exposure. The sequence is performed twice.

\section{(4). Aerobic Recovery Range Section}

Following anaerobic operation conditions, the sensor is exposed to normal air and is subjected to two sequential exposures of $1 \%$ hydrogen. The sensor is allowed to recover after each hydrogen exposure.

All Oxygen Dependence Tests are performed under the following conditions:

Temperature:

Pressure:

Relative Humidity:

Gas Flow Rate: $25^{\circ} \pm 2^{\circ} \mathrm{C}$

$1.0 \pm 0.05$ bar

$<5 \%$ (dry)

$1000 \pm 20 \mathrm{sccm}$ 


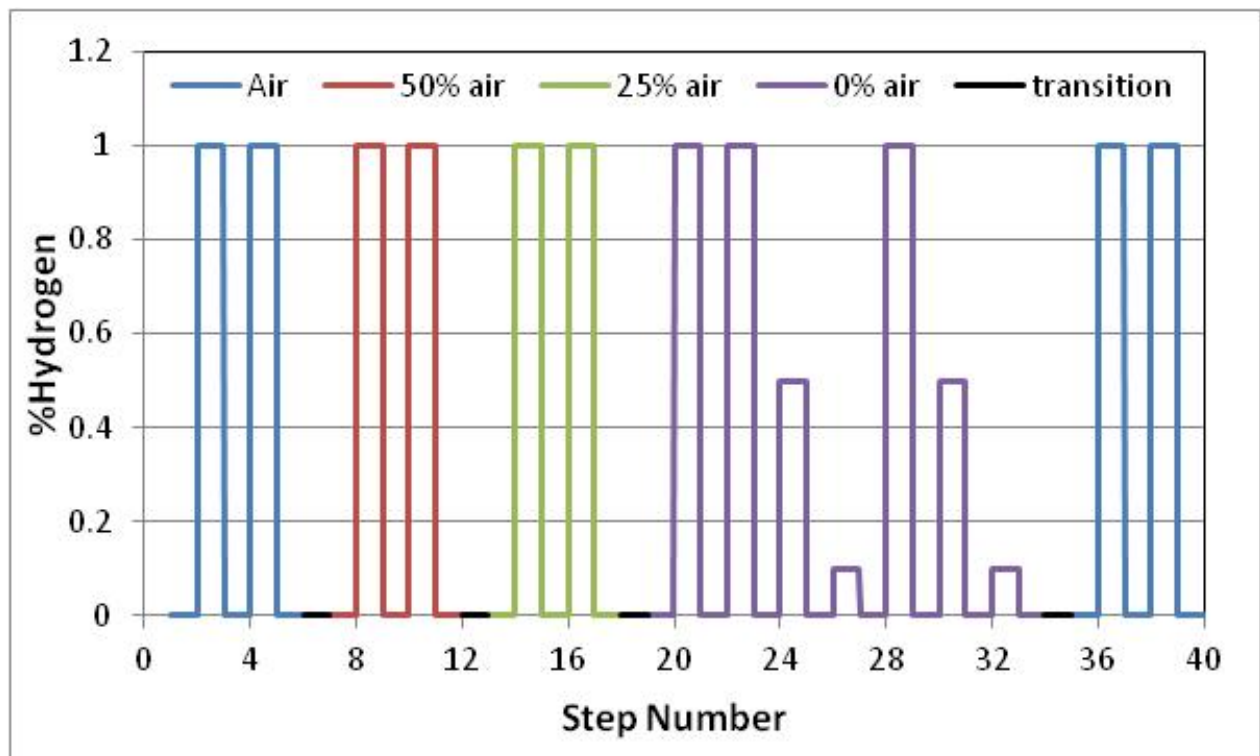

Figure 8: Oxygen Dependence Test. Each sensor is subjected to two sequential exposures of $1 \%$ hydrogen in air followed by recovery in air. The exposure profile is repeated for $50 \%$ air and $25 \%$ air atmospheres. The profile is modified for $0 \%$ air with exposures at $0.5 \%, 0.1 \%$, and $0.05 \%$ hydrogen. Following anaerobic operation, the initial test sequence in air is repeated. The standard duration of each step is one hour. 


\section{Extended Range Test}

The Extended Range Test assesses the ability of a sensor to accurately monitor hydrogen concentrations greater than the LFL and recover from such exposures. Test gas concentrations are $2 \%, 4 \%, 6 \%$, and $8 \%$ hydrogen in a $20 \%$ air ( $4.18 \%$ oxygen). Mixtures of nitrogen and air are used to generate the $20 \%$ air matrix. The depressed oxygen concentration is used for reasons of safety; $8 \%$ hydrogen is below the LFL at $4.75 \%$ oxygen. All Extended Range Tests are performed under the following conditions:

Gas matrix:

Temperature:

Pressure:

Relative Humidity:

Gas Flow Rate:
$20 \%$ air (4.18\% oxygen)

Variable to the designated set point $\pm 2^{\circ} \mathrm{C}$

$1.0 \pm 0.05$ bar

$50 \% \pm 5 \%$

$1000 \pm 20 \mathrm{sccm}$

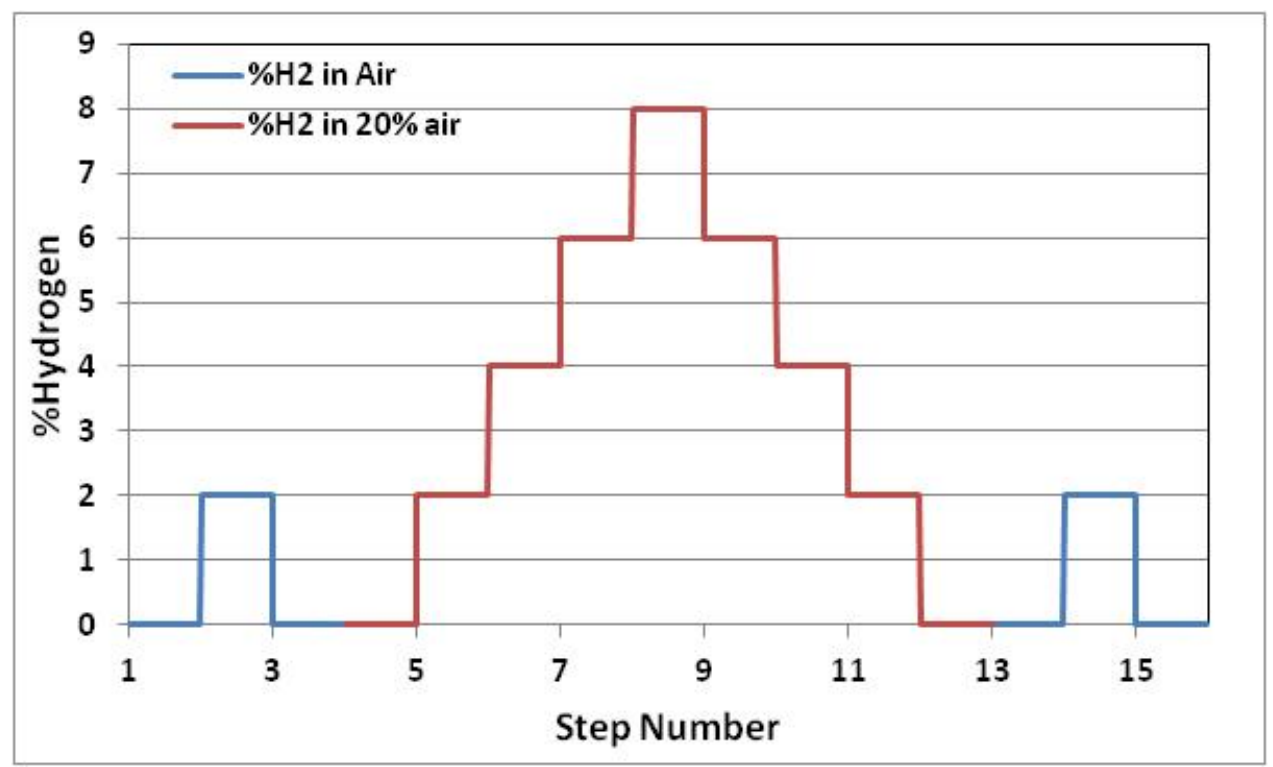

Figure 9: Extended Range Test: Each sensor is exposed to 1\% hydrogen in air and then subjected to a series of hydrogen exposures in $20 \%$ air ( $4.18 \%$ oxygen). Test gas concentrations are $0 \%, 2 \%, 4 \%, 6 \%$, and $8 \%$ hydrogen in ascending and descending levels. Following the extended range test, the sensor is exposed to air and $1 \%$ hydrogen in air. The duration of each step is one hour. 


\section{Long Term Stability Test (Life Test)}

The Long Term Stability Test was developed to evaluate the stability of the test sensor with respect to the potential of long term degradation effects. The sensor is mounted in a dedicated 15 to 20 liter chamber optionally instrumented with temperature and humidity probes (multiple hydrogen sensors may be installed in the same chamber). The internal environment of the chamber is maintained at ambient temperature (regulated by the laboratory HVAC system at $23^{\circ} \pm 3^{\circ} \mathrm{C}$ ), ambient pressure (approximately 0.82 bar for Golden, Colorado), and at $44 \% \pm 5 \% \mathrm{RH}$ (maintained by an internal constant humidity salt). The laboratory $\mathrm{T}, \mathrm{P}$, and $\mathrm{RH}$ are continuously monitored. The internal chamber temperature and $\mathrm{RH}$ are continuously monitored as well. Typically, every two weeks the sensor is exposed to $2 \%$ hydrogen in air. Exposure is performed by purging the chamber with the $2 \%$ hydrogen in air test gas at $2 \mathrm{~L} / \mathrm{min}$ for $45-60$ minutes. Test conditions are maintained at the standard laboratory conditions of $23^{\circ} \pm 2^{\circ} \mathrm{C}, 44 \% \pm 5 \% \mathrm{RH}$, and ambient pressure. Following hydrogen exposure, the chamber is purged with air, which is also maintained at the standard laboratory conditions of $23^{\circ} \pm 2^{\circ} \mathrm{C}$, $44 \% \pm 5 \% \mathrm{RH}$. During the hydrogen exposure, the sensor output is logged at $1 \mathrm{~Hz}$ (i.e., 1 data point per second) for 45-60 minutes prior to the hydrogen purge, during the 45-60 minute hydrogen purge, and for 45-60 minutes of air purge. No further air exchange is performed between hydrogen challenges. The sensor response to the hydrogen challenge is plotted. Also plotted is the final indication versus week of the test. Although the chamber may be opened for maintenance or other purposes, for the most part, it remains sealed for the duration of the Life Test. The Life Test is maintained for a minimum of 3 months (approximately 12 weeks), but is typically performed for longer times. The sensor remains powered on for the duration of the Life Test.
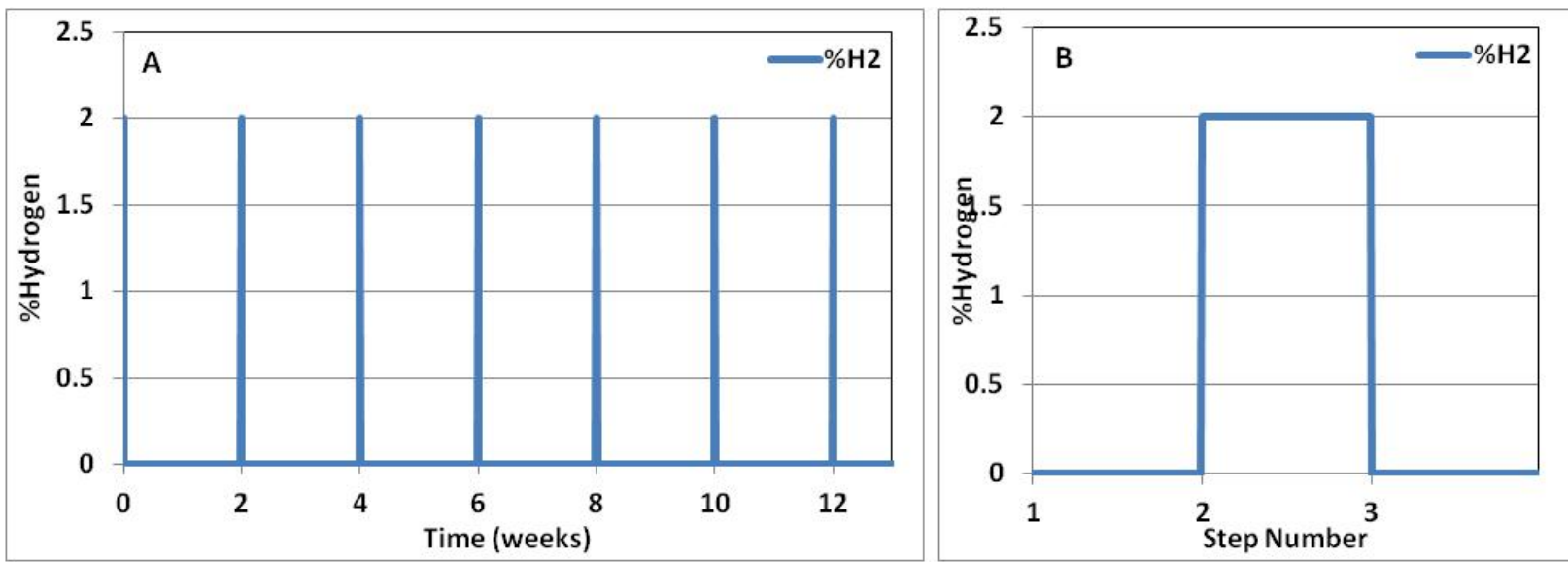

Figure 10: Long Term Stability Test (Life Test) protocol. (A) Each sensor is mounted into a dedicated Life Test chamber purged with clean air and regulated at $44 \% \pm 5 \% \mathrm{RH}$, ambient laboratory temperature and pressure. (B): Every two weeks the sensor is exposed to $2 \%$ hydrogen. During the exposure, the standby environmental conditions $\left(23^{\circ} \pm 3^{\circ} \mathrm{C}, 0.82 \mathrm{bar}, 44 \% \pm 5 \% \mathrm{RH}\right)$ are maintained and the sensor response is recorded for $45-60$ minutes prior to the hydrogen purge (step 1), during the $2 \%$ hydrogen purge (step 2), and during the air purge following the hydrogen exposure (step 3). 
National Renewable Energy Laboratory

1617 Cole Boulevard, Golden, Colorado 80401

303-275-3000 • www.nrel.gov

NREL is a national laboratory of the U.S. Department of Energy

Office of Energy Efficiency and Renewable Energy

Operated by the Alliance for Sustainable Energy, LLC

NREL/BR-5600-53079 • December 2011

Printed with a renewable-source ink on paper containing at least $50 \%$ wastepaper, including $10 \%$ post consumer waste.

NREL is a national laboratory of the U.S. Department of Energy, Office of Energy Efficiency and Renewable Energy, operated by the Alliance for Sustainable Energy, LLC. 